Yufen Zhenga, Ying Zhanga, Hongbo Chia , Shiyong Chen, Minfei Peng, Lifei Luo, Linping Chen, Jun Li, Bo Shen and Donglian Wang*

\title{
The hemocyte counts as a potential biomarker for predicting disease progression in COVID-19: a retrospective study
}

https://doi.org/10.1515/cclm-2020-0377

Received March 25, 2020; accepted April 2, 2020; previously published online April 29, 2020

\section{Abstract}

Objectives: In December 2019, there was an outbreak of coronavirus disease 2019 (COVID-19) in Wuhan, China, and since then, the disease has been increasingly spread throughout the world. Unfortunately, the information about early prediction factors for disease progression is relatively limited. Therefore, there is an urgent need to investigate the risk factors of developing severe disease. The objective of the study was to reveal the risk factors of developing severe disease by comparing the differences in the hemocyte count and dynamic profiles in patients with severe and non-severe COVID-19.

Methods: In this retrospectively analyzed cohort, 141 confirmed COVID-19 patients were enrolled in Taizhou Public Health Medical Center, Taizhou Hospital, Zhejiang Province, China, from January 17, 2020 to February 26, 2020. Clinical characteristics and hemocyte counts of severe and non-severe COVID patients were collected. The differences in the hemocyte counts and dynamic profiles in patients with severe and non-severe COVID-19 were compared. Multivariate Cox regression analysis was performed to identify potential biomarkers for predicting disease progression. A concordance index (C-index), calibration curve, decision curve and the clinical impact curve were calculated to assess the predictive accuracy.

Results: The data showed that the white blood cell count, neutrophil count and platelet count were normal on the day of hospital admission in most COVID-19 patients

\footnotetext{
aYuen Zheng, Ying Zhang and Hongbo Chi contributed equally to this work.

*Corresponding author: Donglian Wang, Department of Clinical Laboratory, Taizhou Hospital, Wenzhou Medical University, Linhai 317000, P.R. China, Phone: +8657685120120 ,

E-mail:wangdl@enzemed.com

Yufen Zheng, Ying Zhang, Hongbo Chi, Shiyong Chen, Minfei Peng, Lifei Luo, Linping Chen, Jun Li and Bo Shen: Department of Clinical Laboratory, Taizhou Hospital, Wenzhou Medical University, Linhai, P.R. China
}

(87.9\%, 85.1\% and $88.7 \%$, respectively). A total of $82.8 \%$ of severe patients had lymphopenia after the onset of symptoms, and as the disease progressed, there was marked lymphopenia. Multivariate Cox analysis showed that the neutrophil count (hazard ratio $[\mathrm{HR}]=4.441,95 \% \mathrm{CI}=1.954-$ 10.090, $\mathrm{p}=0.000)$, lymphocyte count $(\mathrm{HR}=0.255,95 \%$ $\mathrm{CI}=0.097-0.669, \mathrm{p}=0.006)$ and platelet count $(\mathrm{HR}=0.244$, 95\% $\mathrm{CI}=0.111-0.537, \mathrm{p}=0.000)$ were independent risk factors for disease progression. The C-index (0.821 [95\% CI, 0.746-0.896]), calibration curve, decision curve and the clinical impact curve showed that the nomogram can be used to predict the disease progression in COVID-19 patients accurately. In addition, the data involving the neutrophil count, lymphocyte count and platelet count (NLP score) have something to do with improving risk stratification and management of COVID-19 patients.

Conclusions: We designed a clinically predictive tool which is easy to use for assessing the progression risk of COVID-19, and the NLP score could be used to facilitate patient stratification management.

Keywords: COVID-19; disease progression; lymphocyte count; neutrophil count; platelet count.

\section{Introduction}

In December 2019, it was reported that atypical pneumonia occurred in Wuhan, Hubei Province, China, and the cause was later determined to be a novel coronavirus now named severe acute respiratory syndrome coronavirus 2 (SARS-CoV-2) [1]. It has become a pandemic and spread nationally as well as to other countries [2].

SARS-CoV-2 is a new type of coronavirus and its nucleic acid sequence is different from that of severe acute respiratory syndrome coronavirus (SARS-CoV) and Middle East respiratory syndrome coronavirus (MERS-CoV) [1, 3]. Most patients with SARS-CoV-2 infection have mild symptoms (such as fever, dry cough, dyspnea, myalgia, fatigue and so on), but in severe cases, the disease can rapidly progress into acute respiratory distress syndrome, septic shock, bleeding, coagulation dysfunction, metabolic acidosis and death [4]. Therefore, how to identify 
if the patients are at high risk, who are likely to benefit from early intervention with supportive therapy, has recently become the focus of intense research efforts. Trial version 5 has shown that the total number of white blood cells in patients' peripheral blood was normal or slightly decreased, and that the lymphocyte count decreased $[4,5]$. However, information about early prediction factors for severe cases is relatively limited and further investigations are needed.

In this study, we compared the differences in the hemocyte count and dynamic profiles in patients with severe and non-severe coronavirus disease 2019 (COVID19) to reveal the risk factors of developing severe disease.

\section{Materials and methods}

\section{Study design and participants}

We performed this retrospective cohort study in Taizhou Public Health Medical Center, Taizhou Hospital, Zhejiang Province, China. From January 17, 2020 to February 26, 2020, we identified 233 patients with suspected COVID-19 symptoms according to the Diagnosis and Treatment Guideline (trial version 5) of China. A suspected COVID19 case was defined as pneumonia that had related epidemiological history and fulfilled two of the three criteria: fever and/or respiratory symptoms; imaging manifestations of pneumonia; low or normal white blood cell count or low lymphocyte count. All patients were kept in quarantine for at least 2 weeks. A total of 144 patients with laboratory-confirmed infections were included in this study. Then, we excluded three patients younger than 18 years. Finally, 141 COVID19 patients were enrolled in this study (Supplementary Figure S1). As of February 26, 94 patients had been discharged from the hospital.

This study obtained approval from the Medical Ethics Committee of Taizhou Hospital, Zhejiang Province, China, and informed consent was obtained from each enrolled subject.

\section{Data collection}

Information about demographics, epidemiological history, clinical symptoms, underlying comorbidities, hemocyte counts, imaging characteristics and days from symptom onset was collected through an electronic medical record system. The count of white blood cell, neutrophil, lymphocyte, monocyte, eosinophil, platelet and red blood cell as well as the level of hemoglobin were detected using XE-2100 automatic hemocyte analyzer (Sysmex, Japan). The lower limit of detection (LoD) and lower limit of quantitation (LoQ) of lymphocyte, monocyte and eosinophil were $0.01 \times 10^{9} / \mathrm{L}$ and $0.10 \times 10^{9} / \mathrm{L}$, respectively.

The date of symptom onset and hospital admission as well as the severity of COVID-19 were also recorded. The onset date was defined as the day when any symptoms were noticed by the patients. The severity of COVID-19 was defined according to the Diagnosis and Treatment Guideline (trial version 5) of China. Severe COVID-19 was designated when the patients had one of the following criteria: (1) respiratory distress, respiratory rate $\geq 30$ beats/min; (2) oxygen saturation $\leq 93 \%$ in the resting state and (3) arterial blood oxygen partial pressure/oxygen concentration $\leq 300 \mathrm{mmHg}(1 \mathrm{mmHg}=0.133 \mathrm{kPa})$.

\section{Follow-up}

In this study, we classified COVID-19 patients into two groups (severe and non-severe). We defined a non-severe patient as one who presented mild or typical manifestations and a severe patient as one who presented severe or critical manifestations according to the aforementioned classification. The outcome of follow-up was the occurrence of severe illness. The end of follow-up time was March 2, 2020.

\section{Statistical analysis}

Categorical variables were described as frequency and percentage, and continuous variables were shown as mean and standard deviation or median and interquartile range (IQR) values as appropriate. To compare the continuous variables for data from different patient groups, an independent t-test was used when the data were normally distributed; otherwise, the Mann-Whitney test was conducted. The categorical variables were compared using the $\chi^{2}$ test and Fisher's exact test as appropriate. Receiver operating characteristic (ROC) curves were used to select optimal cut-off points. The Cox proportional hazards regression model was used to predict the disease progress. Based on the results of multivariate analysis, a nomogram was established. The nomogram used biological and clinical variables to determine a statistical prognostic model that generates a probability of a clinical event for a particular individual. The concordance index (C-index), calibration curve, decision curve and the clinical impact curve were applied to verify the nomogram. The $\mathrm{C}$-index measured the probability of concordance between predicted and observed survival, ranged from 0.5 to 1.0 , with 0.5 indicating that the outcomes were completely random and 1.0 indicating the perfect discrimination. Decision curves estimated the clinical usefulness of prediction models on the basis of the threshold probability. The clinical impact curve showed the estimated number who would be declared high risk for each risk threshold and visually showed the proportion of those who were cases (true positives) [6, 7]. Kaplan-Meier curves were drawn, and risk stratification between the groups was compared using the log-rank test. All statistical analyses were performed using SPSS version 22.0, R program (version 3.6.0) and GraphPad Prism software (version 8.0). p-Values $<0.05$ were considered statistically significant.

\section{Results}

\section{Clinical characteristics}

The clinical characteristics of the 141 confirmed COVID19 cases, grouped into 112 non-severe cases and 29 severe cases, are shown in Table 1 . The median age was 47 years (IQR, 38-56 years); 100 (70.9\%) patients had histories of exposure in Wuhan; chest CT findings of 134 patients 
Table 1: Clinical characteristics of COVID-19 patients.

\begin{tabular}{|c|c|c|c|c|}
\hline Characteristics & $\begin{array}{r}\text { Total } \\
(n=141)\end{array}$ & $\begin{array}{r}\text { Non-severe } \\
(n=112)\end{array}$ & $\begin{array}{r}\text { Severe } \\
(n=29)\end{array}$ & p-Value \\
\hline Sex, n (\%) & & & & 0.745 \\
\hline Male & $74(52.4)$ & $58(51.7)$ & $16(55.2)$ & \\
\hline Female & $67(47.6)$ & $54(48.3)$ & $13(44.8)$ & \\
\hline Median age (IQR), years & $47(38-56)$ & $45(37-55)$ & $55(47-63)$ & 0.005 \\
\hline First symptom onset to admission, days & $5(3-8)$ & $5(3-8)$ & $7(3-10)$ & 0.242 \\
\hline Wuhan exposure, $n(\%)$ & $100(70.9)$ & $83(74.1)$ & $17(58.6)$ & 0.102 \\
\hline Chest CT, n (\%) & & & & 0.673 \\
\hline Multiple mottled and ground glass opacities & $134(95.0)$ & $106(94.6)$ & $28(96.6)$ & \\
\hline Normal & $7(5.0)$ & $6(5.4)$ & $1(3.4)$ & \\
\hline \multicolumn{5}{|l|}{ Clinical symptoms, n (\%) } \\
\hline Fever & $98(69.5)$ & $73(65.2)$ & $25(86.2)$ & 0.028 \\
\hline Cough & $76(53.9)$ & $59(52.7)$ & $17(58.6)$ & 0.567 \\
\hline Fatigue & $20(14.2)$ & $16(14.3)$ & $4(13.8)$ & 0.946 \\
\hline Dizziness & $10(7.1)$ & $6(5.4)$ & $4(13.8)$ & - \\
\hline Chest tightness & $5(3.5)$ & $5(4.5)$ & $0(0.0)$ & - \\
\hline Diarrhea & $8(5.7)$ & $6(5.4)$ & $2(6.9)$ & 0.749 \\
\hline Underlying comorbidities, n (\%) & $69(48.9)$ & $58(51.8)$ & $11(37.9)$ & 0.183 \\
\hline Clinical outcome & & & & 0.556 \\
\hline Remained in hospital & $47(33.3)$ & $36(32.1)$ & $11(37.9)$ & \\
\hline Discharged & $94(66.7)$ & $76(67.9)$ & $18(62.1)$ & \\
\hline
\end{tabular}

COVID-19, coronavirus disease 2019.

(95.0\%) showed multiple mottled and ground opacities; fever was present in $98(69.5 \%)$ patients and cough in 76 (53.9\%) patients, which were the most common symptoms; $69(48.9 \%)$ patients had underlying comorbidities; and through 26 February, 94 (66.7\%) patients had been discharged, and 47 (33.3\%) patients remained in the hospital. The median age of severe patients was significantly older than that of non-severe patients ( 55 years vs. 45 years, $\mathrm{p}<0.05)$. Compared with non-severe patients, severe patients were more likely to have a fever $(86.2 \%$ vs. $65.2 \%, \mathrm{p}<0.05)$.

\section{Comparison of hemocyte counts between severe and non-severe COVID-19 patients}

It was shown that the white blood cell count of COVID-19 patients on the day of hospital admission was normal in most of the 124 patients (87.9\%). In 120 (85.1\%) COVID-19 patients, the neutrophil count was normal. Lymphopenia was common in all patients $(66,46.8 \%)$, and more patients had lymphopenia in the severe group $(24,82.8 \%)$ than in the non-severe group $(42,37.5 \%, \mathrm{p}=0.000)$. Almost all patients $(134,95.0 \%)$ showed eosinophil count less than $0.10 \times 10^{9} / \mathrm{L}$, and more patients had eosinophil count less than $0.01 \times 10^{9} / \mathrm{L}$ in the severe group $(23,79.3 \%)$ than in the non-severe group $(45,40.2 \%, p=0.001)$. The platelet count was normal in most of the patients $(125,88.7 \%)$, and compared with non-severe patients, severe patients had lower platelet levels $(\mathrm{p}=0.007)$ (as shown in Table 2).

\section{Dynamic profile of hemocyte counts in COVID-19 patients}

To determine the dynamic hemocyte count profile that appeared during COVID-19 progression, we had been following up for more than 27 days since the onset of the first symptom at 3-day intervals. As of February 26, 2020, data from 94 discharged patients (18 severe and 76 non-severe patients) were analyzed (Figure 1). The white blood cell count and neutrophil count increased from the beginning of the disease, reached the highest level at 15-18 days and then decreased gradually. Remarkably, the white blood cell count and neutrophil count were higher in severe than in non-severe patients, but they were still within the normal reference range. In severe patients, there was marked lymphopenia as the disease progressed, and the minimum level appeared on the 12th day, and then it gradually returned to the normal level. However, among non-severe patients, lymphocyte counts were basically normal. The monocyte count was higher in the non-severe group than in the severe group. As the disease progressed and clinical status deteriorated, the eosinophil count remained at 
Table 2: Hemocyte counts of COVID-19 patients on admission to hospital.

\begin{tabular}{|c|c|c|c|c|c|}
\hline & \multirow[t]{2}{*}{ Normal range } & \multicolumn{3}{|r|}{ Median (IQR) } & \multirow[t]{2}{*}{ p-Valu } \\
\hline & & Total $(n=141)$ & Non-severe $(n=112)$ & Severe $(n=29)$ & \\
\hline White blood cell count, $\times 10^{9} / \mathrm{L}$ & $3.5-9.5$ & $5.0(4.2-6.4)$ & $4.9(4.1-6.3)$ & $6.1(4.6-7.8)$ & 0.022 \\
\hline Increased, n (\%) & & $9(6.4)$ & $7(6.3)$ & $2(6.9)$ & 0.000 \\
\hline Normal, n (\%) & & $124(87.9)$ & $98(87.4)$ & $26(89.7)$ & \\
\hline Decreased, n (\%) & & $8(5.7)$ & $7(6.3)$ & $1(3.4)$ & \\
\hline Neutrophil count, $\times 10^{9} / \mathrm{L}$ & $1.8-6.3$ & $3.4(2.5-4.8)$ & $3.2(2.4-4.4)$ & $5.1(3.3-6.7)$ & 0.000 \\
\hline Increased, n (\%) & & $16(11.3)$ & $8(7.1)$ & $8(27.6)$ & 0.000 \\
\hline Normal, n (\%) & & $120(85.1)$ & $100(89.3)$ & $20(69.0)$ & \\
\hline Decreased, n (\%) & & $5(3.6)$ & $4(3.6)$ & $1(3.4)$ & \\
\hline Lymphocyte count, $\times 10^{9} / \mathrm{L}$ & $1.1-3.2$ & $1.1(0.8-1.5)$ & $1.3(0.9-1.6)$ & $0.7(0.4-09)$ & 0.000 \\
\hline Normal, n (\%) & & $75(53.2)$ & $70(62.5)$ & $5(17.2)$ & 0.000 \\
\hline Decreased, n (\%) & & $66(46.8)$ & $42(37.5)$ & $24(82.8)$ & \\
\hline Monocyte count, $\times 10^{9} / \mathrm{L}$ & $0.1-0.6$ & $0.4(0.3-0.5)$ & $0.4(0.3-0.5)$ & $0.3(0.2-0.5)$ & 0.011 \\
\hline Increased, n (\%) & & $15(10.6)$ & $13(11.6)$ & $2(6.9)$ & 0.693 \\
\hline Normal, n (\%) & & $126(89.4)$ & $99(88.4)$ & $27(93.1)$ & \\
\hline Eosinophil count, $\times 10^{9} / \mathrm{L}$ & & & & & 0.001 \\
\hline$<0.01, \mathrm{n}(\%)$ & & $68(48.2)$ & $45(40.2)$ & $23(79.3)$ & \\
\hline $0.01-0.10, \mathrm{n}(\%)$ & & $66(46.8)$ & $61(54.5)$ & $5(17.2)$ & \\
\hline$>0.10$, n. (\%) & & $7(5.0)$ & $6(5.3)$ & $1(3.5)$ & \\
\hline Platelet count, $\times 10^{9} / \mathrm{L}$ & $125-350$ & $199(160-240)$ & $203(169-246)$ & $158(125-222)$ & 0.007 \\
\hline Increased, n (\%) & & $4(2.8)$ & $4(3.6)$ & $0(0.0)$ & 0.000 \\
\hline Normal, n (\%) & & $125(88.7)$ & $103(92.0)$ & $22(75.9)$ & \\
\hline Decreased, n (\%) & & $12(8.5)$ & $5(4.4)$ & $7(24.1)$ & \\
\hline $\begin{array}{l}\text { Red blood cell count, } \times 10^{12} / \mathrm{L} \\
\text { (mean } \pm \text { SD) }\end{array}$ & $\begin{array}{l}\text { Male: } 4.3-5.8 \\
\text { female: } 3.8-5.1\end{array}$ & $4.6 \pm 0.5$ & $4.6 \pm 0.5$ & $4.6 \pm 0.5$ & 0.894 \\
\hline Increased, n (\%) & & $5(3.6)$ & $4(3.6)$ & $1(3.4)$ & 1.000 \\
\hline Normal, n (\%) & & $123(87.2)$ & $97(86.6)$ & $26(89.7)$ & \\
\hline Decreased, n (\%) & & $13(9.2)$ & $11(9.8)$ & $2(6.9)$ & \\
\hline Hemoglobin, g/L & $\begin{array}{l}\text { Male: } 130-175 \\
\text { female: } 115-150\end{array}$ & $137(128-151)$ & $128(137-152)$ & $135(127-151)$ & 0.713 \\
\hline Increased, n (\%) & & $3(2.1)$ & $3(2.7)$ & $0(0.0)$ & 1.000 \\
\hline Normal, n (\%) & & $122(86.5)$ & $98(87.5)$ & $24(82.8)$ & \\
\hline Decreased, n (\%) & & $16(11.4)$ & $11(9.8)$ & $5(17.2)$ & \\
\hline
\end{tabular}

According to the LoD and LoQ of eosinophil count, COVID-19 patients were divided into three groups $\left(<0.01 \times 10^{9} / \mathrm{L}, 0.01-0.10 \times 10^{9} / \mathrm{L}\right.$, $>0.10 \times 10^{9} /$ L, respectively). COVID-19, coronavirus disease 2019; LoD, lower limit of detection; LoQ, lower limit of quantitation.

extremely low levels during the first 18 days, but as the patients got better, the eosinophil count increased less in the severe group than in the non-severe group (18-24 days) $(p<0.05)$. The platelet count was higher in the non-severe group than in the severe group $(\mathrm{p}<0.05)$.

Similarly, we compared the differences in blood cell counts between initial onset and convalescence in 94 patients discharged from the hospital. The initial onset data were from the test results on the day of or the day after admission, and the convalescence data were from the test results 1-2 days before discharge (Figure 2). In the early stage of the disease, the white blood cell $(\mathrm{p}<0.01)$ and neutrophil counts $(p<0.0001)$ were higher in the severe patients than in the non-severe patients at initial onset, while the lymphocyte count was reduced in the severe patients, and that in the non-severe patients was basically normal $(p<0.001)$. The eosinophil count was generally very low in the early stages of the disease in severe patients, and returned to normal levels upon convalescence $(p<0.001)$.

\section{Predictive disease progression value of hemocyte counts}

We then investigated the ability of the hemocyte count to predict the progression of severe disease. The results of univariate and multivariate Cox proportional hazards regression analyses of predictive factors for disease progress are shown in Table 3. Multivariate analysis showed that the neutrophil count (hazard ratio $[\mathrm{HR}]=4.441$, 95\% CI $=1.954-10.090, \mathrm{p}=0.000$ ), lymphocyte count $(\mathrm{HR}=0.255,95 \% \mathrm{CI}=0.097-0.669, \mathrm{p}=0.006)$ and platelet count $(\mathrm{HR}=0.244,95 \% \mathrm{CI}=0.111-0.537, \mathrm{p}=0.000)$ were 

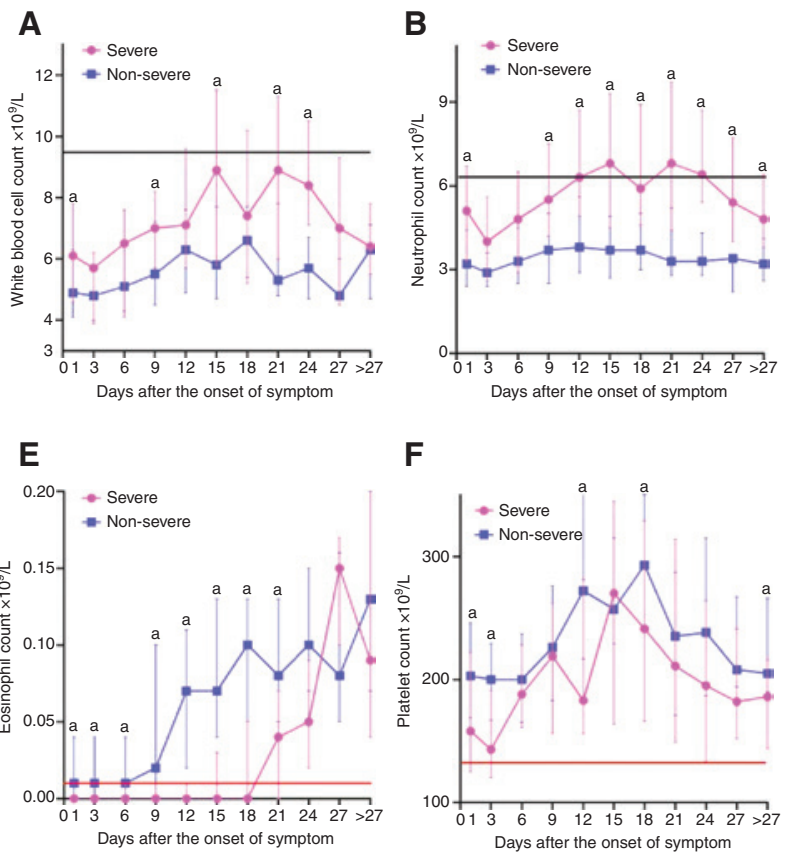

$\mathbf{F}$

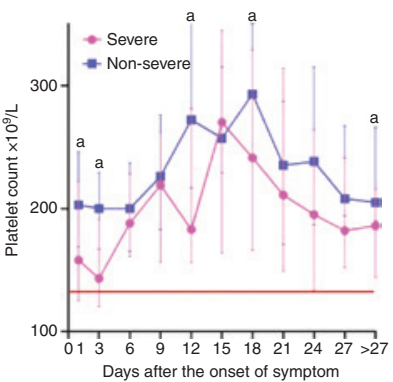

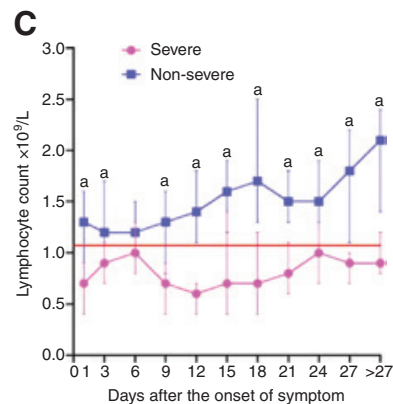

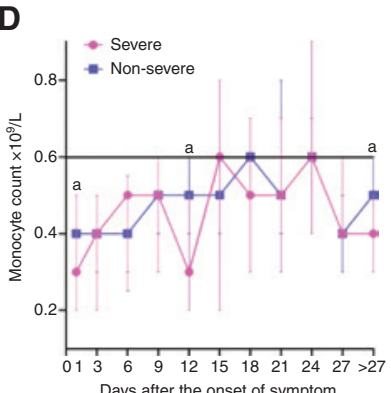

G

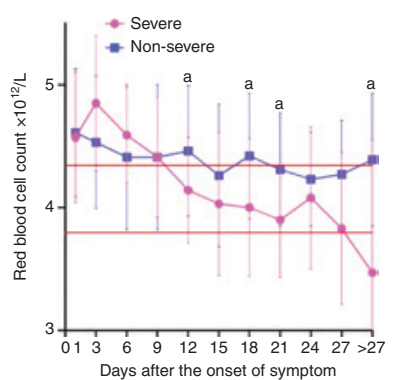

H

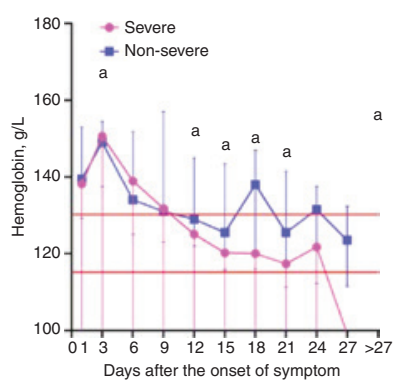

Figure 1: Timeline charts illustrating the dynamic profiles of hemocyte counts among 94 discharged patients (18 severe and 76 non-severe patients) at 3-day intervals based on the days after the first symptom onset.

The solid lines in black show the upper normal limit of the white blood cell count, neutrophil count and monocyte count; the solid lines in red show the lower normal limit of the lymphocyte count, eosinophil count, platelet count, red blood cell count and hemoglobin. ${ }^{a} p<0.05$ for non-severe vs. severe patients.
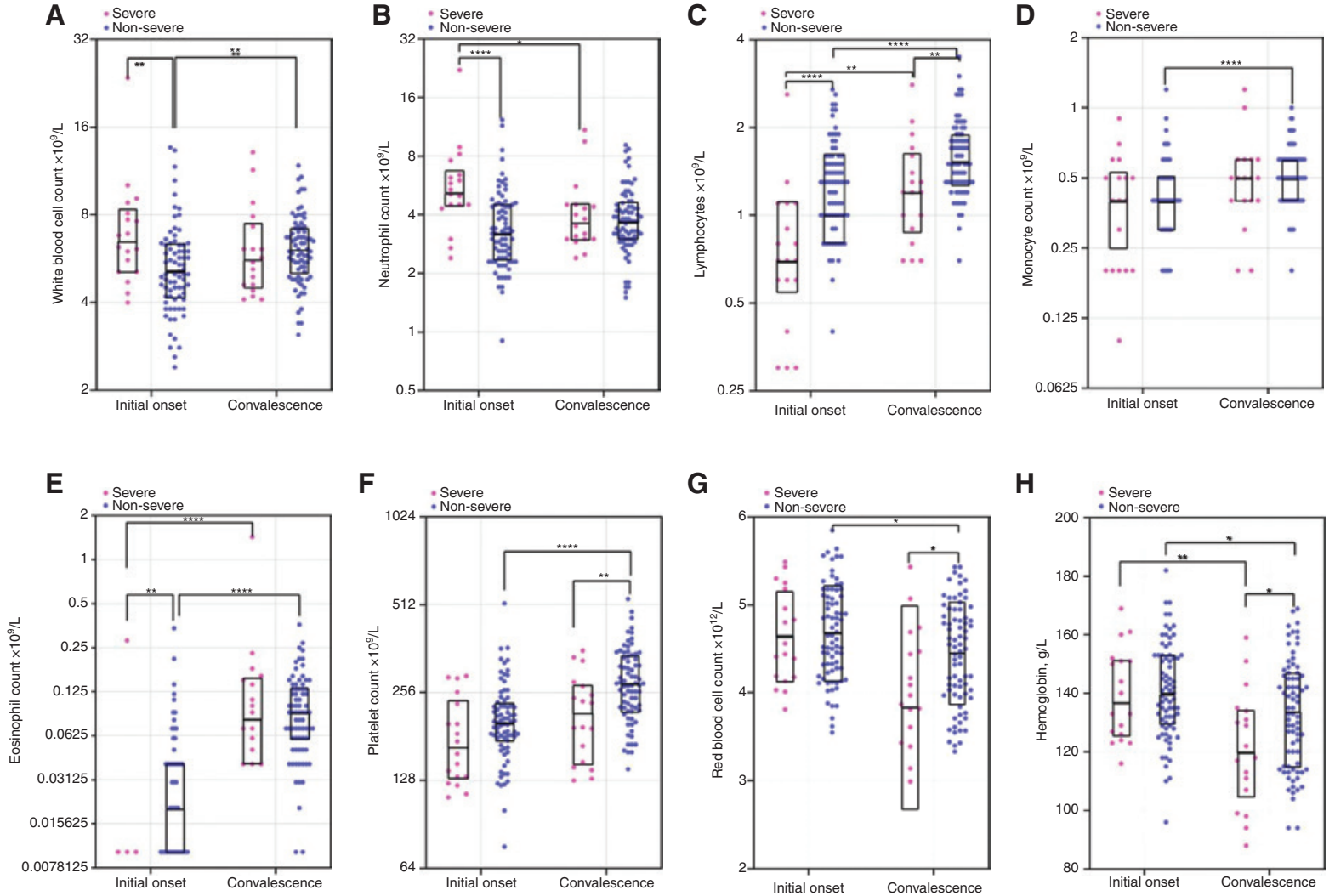

Figure 2: Scatter plot of hemocyte counts at initiation onset and at convalescence in 94 patients discharged from the hospital. ${ }^{\star} p<0.05,{ }^{* \star} p<0.01,{ }^{* \star *} p<0.001$ and ${ }^{\star \star \star *} p<0.0001$. 
Table 3: Univariate and multivariate Cox regression analyses of disease progression in COVID-19 patients

\begin{tabular}{|c|c|c|c|c|}
\hline Variates & $\begin{array}{r}\text { Univariate analysis } \\
\mathrm{HR}(95 \% \mathrm{Cl})\end{array}$ & p-Value & $\begin{array}{r}\text { Multivariate analysis } \\
\mathrm{HR}(95 \% \mathrm{Cl})\end{array}$ & p-Value \\
\hline Sex & & 0.833 & & \\
\hline Male & 1 & & & \\
\hline Female & $0.886(0.289-2.719)$ & & & \\
\hline Age, years & & 0.001 & & 0.185 \\
\hline$\leq 50$ & 1 & & 1 & \\
\hline$>50$ & $3.826(1.694-8.645)$ & & $1.022(0.990-1.054)$ & \\
\hline Fever & & 0.037 & & 0.098 \\
\hline No & 1 & & 1 & \\
\hline Yes & $3.075(1.070-8.838)$ & & $2.509(0.843-7.463)$ & \\
\hline Cough & & 0.660 & & \\
\hline No & 1 & & & \\
\hline Yes & $1.181(0.564-2.472)$ & & & \\
\hline Fatigue & & 0.839 & & \\
\hline No & 1 & & & \\
\hline Yes & $0.896(0.312-2.576)$ & & & \\
\hline Dizziness & & 0.094 & & \\
\hline No & 1 & & & \\
\hline Yes & $2.467(0.858-7.097)$ & & & \\
\hline Diarrhea & & 0.741 & & \\
\hline No & 1 & & & \\
\hline Yes & $1.274(0.303-5.360)$ & & & \\
\hline Underlying comorbidities & & 0.181 & & \\
\hline No & 1 & & & \\
\hline Yes & $0.599(0.283-1.269)$ & & & \\
\hline Wuhan exposure & & 0.054 & & 0.329 \\
\hline No & 1 & & 1 & \\
\hline Yes & $0.438(0.189-1.016)$ & & $0.535(0.152-1.878)$ & \\
\hline Chest CT & & 0.672 & & 0.609 \\
\hline Normal & 1 & & 1 & \\
\hline Multiple mottled and ground glass opacities & $1.538(0.209-11.302)$ & & $0.583(0.073-4.629)$ & \\
\hline White blood cell count, $\times 10^{9} / \mathrm{L}$ & & 0.006 & & 0.438 \\
\hline$\leq 5.95$ & 1 & & 1 & \\
\hline$>5.95$ & $2.809(1.341-5.884)$ & & $1.980(0.352-11.135)$ & \\
\hline Neutrophil count, $\times 10^{9} / \mathrm{L}$ & & 0.000 & & 0.000 \\
\hline$\leq 4.45$ & 1 & & 1 & \\
\hline$>4.45$ & $4.878(2.266-10.501)$ & & $4.441(1.954-10.090)$ & \\
\hline Lymphocyte count, $\times 10^{9} / \mathrm{L}$ & & 0.000 & & 0.006 \\
\hline$\leq 0.95$ & 1 & & 1 & \\
\hline$>0.95$ & $0.122(0.050-0.300)$ & & $0.255(0.097-0.669)$ & \\
\hline Monocyte count, $\times 10^{9} / \mathrm{L}$ & & 0.000 & & 0.081 \\
\hline$\leq 0.25$ & 1 & & 1 & \\
\hline$>0.25$ & $0.230(0.110-0.483)$ & & $0.488(0.218-1.093)$ & \\
\hline Eosinophil count, $\times 10^{9} / \mathrm{L}$ & & 0.001 & & 0.188 \\
\hline$\leq 0.005$ & 1 & & 1 & \\
\hline$>0.005$ & $0.212(0.086-0.522)$ & & $0.510(0.188-1.388)$ & \\
\hline Platelet count, $\times 10^{9} / \mathrm{L}$ & & 0.000 & & 0.000 \\
\hline$\leq 158.5$ & 1 & & 1 & \\
\hline$>158.5$ & $9.068(4.114-19.987)$ & & $0.244(0.111-0.537)$ & \\
\hline Hemoglobin, $\mathrm{g} / \mathrm{L}$ & & 0.441 & & \\
\hline$\leq 134.5$ & 1 & & & \\
\hline$>134.5$ & $0.751(0.362-1.556)$ & & & \\
\hline Red blood cell count, $\times 10^{12} / \mathrm{L}$ & & 0.340 & & \\
\hline$\leq 4.6$ & 1 & & & \\
\hline$>4.6$ & $0.694(0.328-1.470)$ & & & \\
\hline
\end{tabular}

COVID-19, coronavirus disease 2019. 
A

Points

Neutrophil

Lymphocyte

Platelet

Total points

7-day non-severe survival

14-day non-severe survival

21-day non-severe surviva

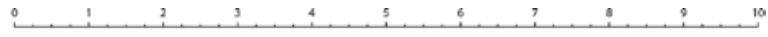

B

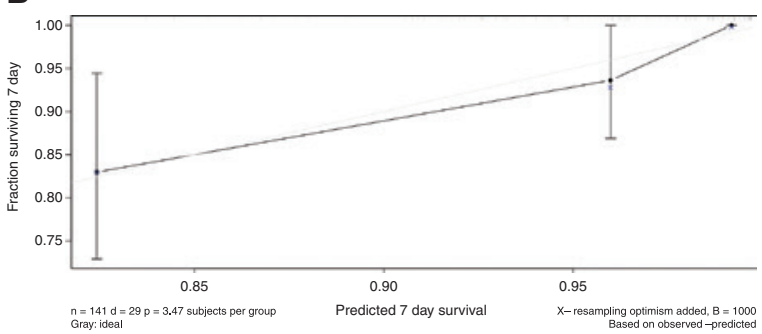

C

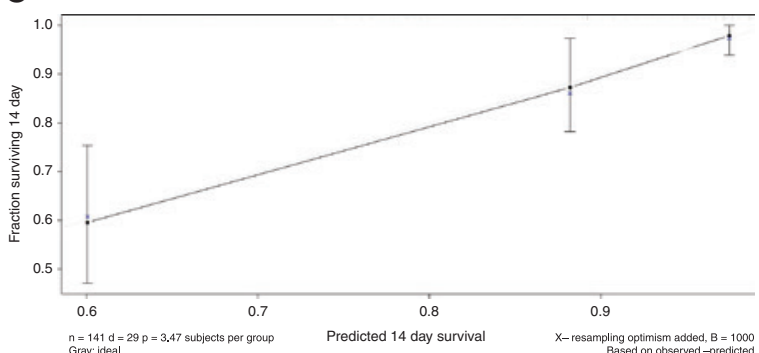

D

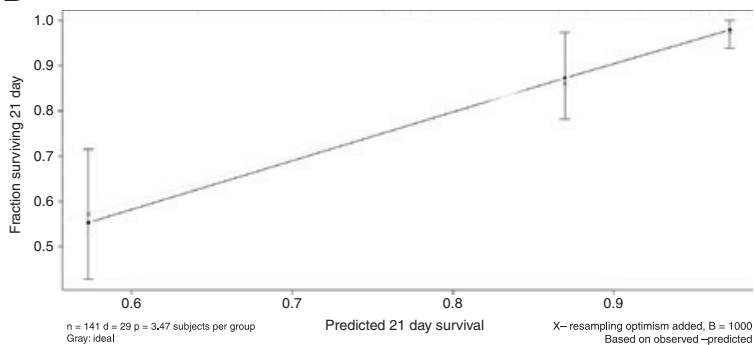

E
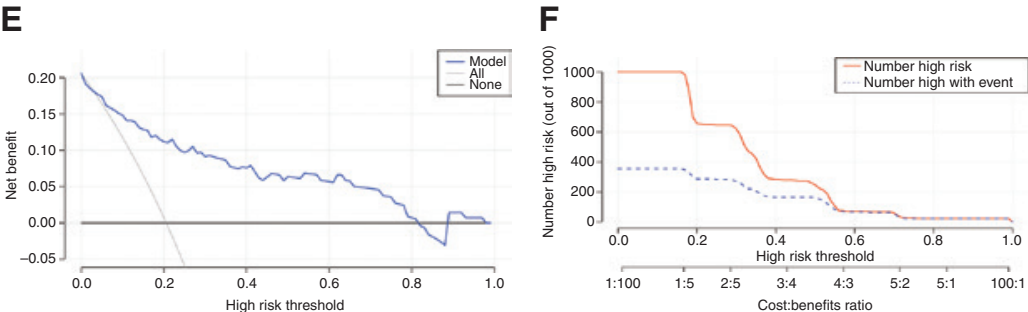

Figure 3: Non-severe COVID-19 patient survival nomogram, calibration curve, decision curve and clinical impact curve.

(A) Nomogram predicting severe COVID-19 patients. To use the nomogram: the value of an individual patient is located on each variable axis, and a line is drawn upward to determine the number of points received for each variable value. The sum of these numbers is located on the total point axis, and a line is drawn downward to the survival axes to determine the likelihood of 7-day, 14-day and 21-day non-severe survival. The calibration curve is used for predicting non-severe survival at 7 days (B), 14 days (C) and 21-days (D). The nomogram-predicted probability of non-severe survival is plotted on the $x$-axis and that of actual non-severe survival is plotted on the $y$-axis. (E) Decision curve: the abscissa of this graph is the threshold probability and the ordinate is the net benefit. (F) Clinical impact curve: the red curve (number of high-risk individuals) indicates the number of people who are classified as positive (high risk) by the model at each threshold probability; the blue curve (number of high-risk individuals with outcome) is the number of true positives at each threshold probability. COVID-19, coronavirus disease 2019. 
independent risk factors for disease progression. ROC curve analysis revealed that the cut-off neutrophil count was $4.45 \times 10^{9} / \mathrm{L}$, lymphocyte count was $0.95 \times 10^{9} / \mathrm{L}$ and platelet count was $158.5 \times 10^{9} / \mathrm{L}$.

\section{Establishment and accuracy prediction of a nomogram}

To individually predict the incidence of disease progression for each COVID-19 patient, we established a novel nomogram that integrated the neutrophil count and lymphocyte count with the platelet count for 7-day non-severe survival, 14-day non-severe survival and 21-day nonsevere survival (Figure 3A). The C-index of the nomogram was 0.821 (95\% CI, 0.746-0.896). The calibration curve represents an internal validation system (Figure 3B-D). It showed considerable consistency between the prediction and actual observation for 7-day non-severe survival, 14-day non-severe survival and 21-day non-severe survival which indicates that the nomogram has a good predictive effect on the disease progression of COVID-19 patients. The decision curve (Figure 3E) and clinical impact curve (Figure 3F) also indicated that the nomogram had good net benefits for the identification of severe COVID-19 patients.

\section{Performance of the neutrophil, lymphocyte and platelet (NLP score) in stratifying the risk of patients}

We designed a clinically predictive tool which is easy to use for assessing the progression risk of COVID-19. A predictive NLP score was calculated on the basis of a multivariate Cox proportional hazards regression model that included the neutrophil count, lymphocyte count and

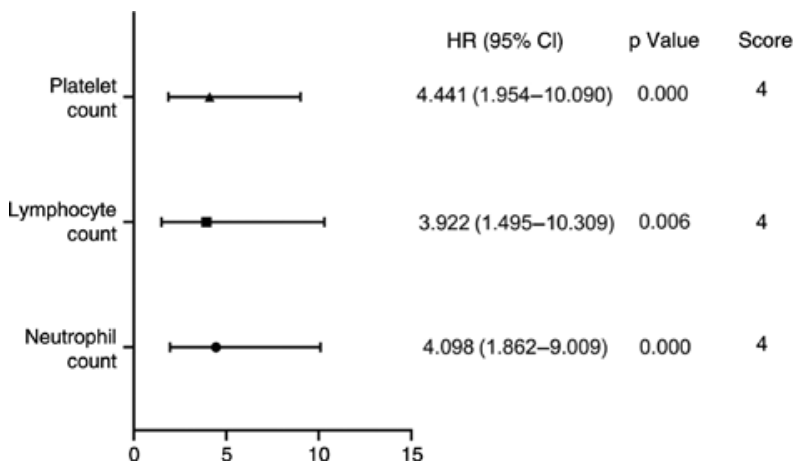

Figure 4: Multivariate analysis associated with the disease progression risk of COVID-19 patients.

COVID-19, coronavirus disease 2019.

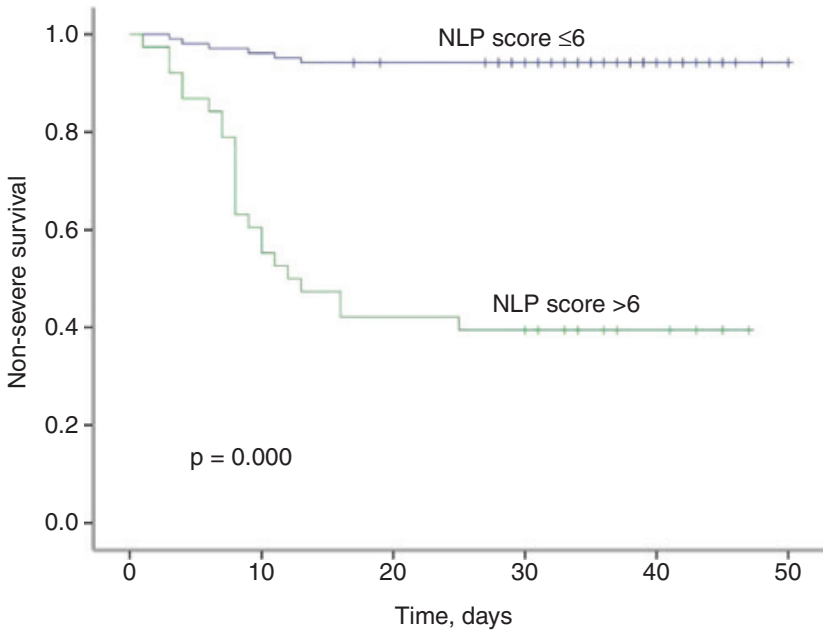

Figure 5: Kaplan-Meier (K-M) curves of disease progression risk stratification for non-severe COVID-19 patients.

For disease progression risk: NLP score $0-6=$ low risk; $>6=$ high risk. COVID-19, coronavirus disease 2019. NLP score $=$ neutrophil count, lymphocyte count and platelet count score.

Table 4: NLP score in COVID-19 patients

\begin{tabular}{lr}
\hline NLP & Score \\
\hline Neutrophil count & \\
$\leq 4.45 \times 10^{9} / \mathrm{L}$ & 0 \\
$<4.45 \times 10^{9} / \mathrm{L}$ & 4 \\
Lymphocyte count & \\
$\leq 0.95 \times 10^{9} / \mathrm{L}$ & 4 \\
$>0.95 \times 10^{9} / \mathrm{L}$ & 0 \\
Platelet count & \\
$\leq 158.5 \times 10^{9} / \mathrm{L}$ & 4 \\
$>158.5 \times 10^{9} / \mathrm{L}$ & 0 \\
\hline
\end{tabular}

NLP score $\leq 6$, the risk of progression to a severe COVID-19 was low; NLP score $>6$, the risk of progression to a severe COVID-19 was high. NLP score, neutrophil count, lymphocyte count and platelet count.

platelet count, and relative weight scores were assigned according to the HR (Figure 4). The optimal cut-off point of the NLP score was calculated using the ROC curve. Patients were divided into high-risk and low-risk groups considering the cut-off value of 6 . Statistically significant differences were observed between the two groups using the Kaplan-Meier analysis ( $p=0.000$, Figure 5$)$. The NLP score is shown in Table 4.

\section{Discussion}

As of 26 February 2020, we reported a cohort of 141 COVID19 patients from Taizhou Public Health Medical Center, Taizhou Hospital, Zhejiang Province. To the best of our 
knowledge, the sample size was the largest outside the Wuhan region, and comprehensive hemocyte counts were investigated in our study. In this study, the most important finding was that the neutrophil count, lymphocyte count and platelet count were independent risk factors for predicting the development of severe illness in COVID19 patients. A prognostic model for severe patients with COVID-19 was established by a nomogram, and the accuracy of the nomogram was demonstrated through calibration curves, decision curves and clinical impact curves, indicating its significant predictive value.

The results of this study showed that the lymphocyte count decreased among nearly half (46.8\%) of the patients in the earliest stage, and $82.8 \%$ of patients with severe disease suffered from lymphocytopenia. Furthermore, the lymphocytopenia was more obvious as the disease progressed, which was consistent with literature reports [8]. Lymphocytopenia is a prominent feature of severe COVID19 patients because targeted invasion by SARS-CoV-2 viral particles damages the cytoplasmic component of the lymphocyte and leads to destruction [9]. Additionally, lymphocytopenia was also common in severe patients with MERS infection, which was the result of apoptosis of lymphocytes $[10,11]$. Therefore, we presumed that necrosis or apoptosis of lymphocytes also induces lymphocytopenia in severe patients with SARS-CoV-2 infection. A previous study showed that among the majority of severe patients infected with SARS-CoV-2, there were $35 \%$ of patients with only mild lymphocytopenia [12], while it was shown that there were $37.5 \%$ of non-severe patients with lymphocytopenia in this study, suggesting that the severity of SARSCoV-2 infection was related to lymphocytopenia.

The results of this study showed that the neutrophil count in patients with severe disease was higher than that in those with non-severe disease, while the platelet count in patients with the severe type was lower than that in those with the non-severe type. However, as the disease progressed, the neutrophil count increased and the platelet count decreased, especially among severe patients. Multivariate analysis showed that the neutrophil count and platelet count were independent risk factors for disease progression. Others have also reported neutrophilia to be a predictor of poor outcome in SARS patients $[13,14]$. Tsui et al. [13] believed that this phenomenon may be a representation of disease progression in SARS patients or a high viral load exposure, or may indicate that the patient will require more supportive treatment. The two reasons mentioned earlier may explain the similar observations we made. Alternatively, neutrophilia may be related to cytokine storms induced by virus invasion [5]. Steroids may cause neutrophilia and they have been used in the treatment of severe SARS patients [15, 16]. Hoheisel et al. [17] reported that thrombocytopenia was the most prominent laboratory finding in SARS patients, reflecting a more severe clinical course. Liu et al. [18] and He et al. [19] reported that thrombocytopenia was closely related to prognosis of SARS patients in multivariate analysis. This finding implied that a decrease in the platelet count, especially a gradual decrease, was a marker of aggravation of illness state and suggested that appropriate emergency measures should be taken immediately to prevent the disease from getting worse.

Interestingly, almost all patients (134, 95.0\%) showed eosinophil count less than $0.10 \times 10^{9} / \mathrm{L}$ in the early stages of the disease, and especially in severe patients, the eosinophil count was generally very low and returned to normal levels upon convalescence. This may be associated with a mechanism of stress response in the condition of acute lung injury caused by SARS-CoV-2, which inhibits the release of eosinophil in the bone marrow through glucocorticoid secretion [20]. As the disease recovers, the eosinophil gradually returns to normal levels, which means that decreasing and increasing eosinophil may be the signs of disease progression and recovery.

According to our results, we speculate that the NLP score may be of help to improve risk stratification and management of COVID-19 patients. Patients can be classified into two groups (Group 1 exhibited an NLP score $\leq 6$; Group 2 exhibited an NLP score $>6$ ). For a higher NLP score, more attention needs to be paid to the progression of COVID-19 patients. Risk stratification and management will be helpful for us to identify severe patients early enough to control disease progression.

However, this study also has some limitations. For one thing, it was a retrospective single center study, so external validation is required in large sample and multicenter studies. For another, some of the patients included were still hospitalized during the study period. Furthermore, the dynamic data were partly missing for some COVID-19 patients.

\section{Conclusions}

In short, despite increasing worldwide efforts to reduce the physical and socioeconomic impact of COVID-19, reliable biomarkers for predicting the disease severity remain scarce. This study provided important insight into the association between the neutrophil count, lymphocyte count and platelet count (NLP score) and risk of disease progression. These results could be used to facilitate patient stratification management. 
Research funding: This study was financially supported by the National Natural Science Foundation of China (Grant No. 81672086).

Author contributions: All authors have accepted responsibility for the entire content of this manuscript and approved its submission.

Competing interests: Authors state no conflict of interest. Informed consent: Informed consent was obtained from all individuals included in this study.

Ethical approval: This study obtained approval from the Medical Ethics Committee of Taizhou Hospital, Zhejiang Province, China.

\section{References}

1. Zhu N, Zhang D, Wang W, Li X, Yang B, Song J, et al. A novel coronavirus from patients with pneumonia in China, 2019. New Engl J Med 2020;382:727-33.

2. Giovanetti M, Benvenuto D, Angeletti S, Ciccozzi M. The first two cases of 2019-nCoV in Italy: where they come from? J Med Virol 2020;92:518-21.

3. Lu R, Zhao X, Li J, Niu P, Yang B, Wu H, et al. Genomic characterisation and epidemiology of 2019 novel coronavirus: implications for virus origins and receptor binding. Lancet 2020;395:565-74.

4. Huang C, Wang Y, Li X, Ren L, Zhao J, Hu Y, et al. Clinical features of patients infected with 2019 novel coronavirus in Wuhan, China. Lancet 2020;395:497-506.

5. Wang D, Hu B, Hu C, Zhu F, Liu X, Zhang J, et al. Clinical characteristics of 138 hospitalized patients with 2019 novel coronavirus-infected pneumonia in Wuhan, China. J Am Med Assoc 2020;7:e201585.

6. Balachandran VP, Gonen M, Smith JJ, DeMatteo RP. Nomograms in oncology: more than meets the eye. Lancet Oncol 2015;16:e173-80.

7. Altman DG, Vergouwe Y, Royston P, Moons KG. Prognosis and prognostic research: validating a prognostic model. Br Med J 2009;338:b605.

8. Yang X, Yu Y, Xu J, Shu H, Liu H, Wu Y, et al. Clinical course and outcomes of critically ill patients with SARS-CoV-2 pneumonia in Wuhan, China: a single-centered, retrospective, observational study. Lancet Respir Med 2020;8:e26.
9. Gu J, Gong E, Zhang B, Zheng J, Gao Z, Zhong Y, et al. Multiple organ infection and the pathogenesis of SARS. J Exp Med 2005;202:415-24.

10. Chu H, Zhou J, Wong BH, Li C, Chan JF, Cheng Z-S, et al. Middle East respiratory syndrome coronavirus efficiently infects human primary $T$ lymphocytes and activates the extrinsic and intrinsic apoptosis pathways. J Infect Dis 2016;213:904-14.

11. Liu WJ, Zhao M, Liu K, Xu K, Wong G, Tan W, et al. T-cell immunity of SARS-CoV: implications for vaccine development against MERS-CoV. Antivir Res 2017;137:82-92.

12. Chen N, Zhou M, Dong X, Qu J, Gong F, Han Y, et al. Epidemiological and clinical characteristics of 99 cases of 2019 novel coronavirus pneumonia in Wuhan, China: a descriptive study. Lancet 2020;395:507-13.

13. Tsui PT, Kwok ML, Yuen H, Lai ST. Severe acute respiratory syndrome: clinical outcome and prognostic correlates. Emerg Infect Dis 2003;9:1064.

14. Lee N, Hui D, Wu A, Chan P, Cameron P, Joynt GM, et al. A major outbreak of severe acute respiratory syndrome in Hong Kong. New Engl J Med 2003;348:1986-94.

15. Tai D, Lew T, Loo S, Earnest A, Chen M, Group SI. Clinical features and predictors for mortality in a designated national SARS ICU in Singapore. Ann Acad Med Singap 2003;32:S34.

16. So LK, Lau AC, Yam LY, Cheung TM, Poon E, Yung RW, et al. Development of a standard treatment protocol for severe acute respiratory syndrome. Lancet 2003;361:1615-7.

17. Hoheisel G, Wu A, Lee N, Chan CH, Wong KT, Ahuja A, et al. [Severe acute respiratory syndrome (SARS)]. Pneumologie (Stuttgart, Germany) 2003;57:315-21.

18. Liu X, Chen S, He G, Li Y, He W, Chen R, et al. Management of critical severe acute respiratory syndrome and risk factors for death. Zhonghua Jie He He Hu Xi Za Zhi 2003;26:329-33.

19. He W, Chen S, Liu X, Li Y, Xiao Z, Zhong N. Death risk factors of severe acute respiratory syndrome with acute respiratory distress syndrome. Zhongguo Wei Zhong Bing Ji Jiu Yi Xue 2003;15:336-7.

20. Liu F, Xu A, Zhang Y, Xuan W, Yan T, Pan K, et al. Patients of COVID-19 may benefit from sustained lopinavir-combined regimen and the increase of eosinophil may predict the outcome of COVID-19 progression. Int J Infect Dis 2020;S1201-9712(20)30132-6. DOI: 10.1016/j.ijid.2020.03.013.

Supplementary Material: The online version of this article offers supplementary material (https://doi.org/10.1515/cclm-2020-0377). 